\title{
DEVELOPMENT OF A COMPACT ELECTRON BEAM FLUORESCENCE INSTRUMENT FOR HIGH ENTHALPY FLOW CHARACTERIZATION
}

\author{
B. Diop, A. Mohamed, and J. Bonnet \\ ONERA \\ BP72, Chatillon Cedex 92322, France
}

\begin{abstract}
A prototype based on the Electron Beam Fluorescence (EBF) Technique is being developed for in-flight measurements in the shock layer of a reentry demonstrator. The measurement objectives are rotational and vibrational temperatures of $\mathrm{N}_{2}$ as well as the densities of $\mathrm{N}_{2}$ and $\mathrm{NO}$ at different positions in and outside a shock layer. There is a renewal of interest in extending the technique to higher densities and to probe $\mathrm{CO} / \mathrm{CO}_{2}$ flows (Mars atmosphere studies). The present paper gives a brief description of the status of the instrument with details on the optimization work performed to miniaturize the electron gun, the study of induced fluorescence in some gases and mixtures in laboratory vacuum chamber.
\end{abstract}

\section{INTRODUCTION}

The characterization of high enthalpy flows is of paramount importance for an improved understanding of various tests and experiments which are conducted in supersonic facilities to simulate the dynamic and energy exchanges which take place during the reentry of vehicles through the upper atmosphere. The EBF has been identified as a good candidate among nonintrusive techniques for in-flight data collection of shock layer chemistry. It can provide direct measurements of flow physical properties to be used at a later stage for validation of aerothermodynamics design tools.

The objectives of this measurement technique in reentry applications is to measure vibrational and rotational temperatures of $\mathrm{N}_{2}$ and partial densities of $\mathrm{N}_{2}$ and $\mathrm{NO}$ in at least two selected locations within the flow-field surrounding a reentry vehicle, for example, one point in the shock layer and one point in a free stream. In this way, the EBF serves also as an air data system as it provides a direct measurement of the free stream density, the knowledge of which is necessary for postflight analysis.

This is an Open Access article distributed under the terms of the Creative Commons Attribution-Noncommercial License 3.0, which permits unrestricted use, distribution, and reproduction in any noncommercial medium, provided the original work is properly cited. 


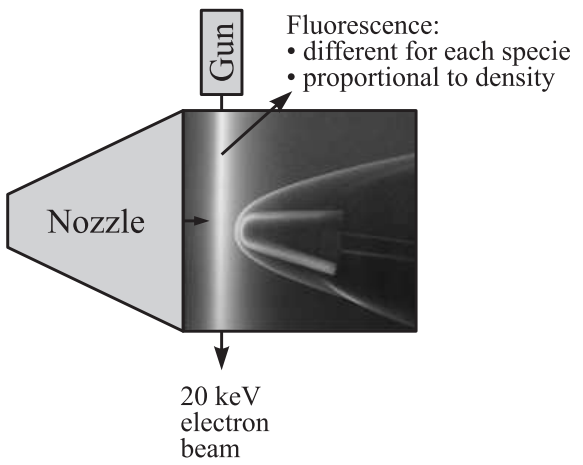

Figure 1 Typical setup of EBF in a windtunnel - visualization of the electron beam and exploitation of its afterglow to visualize a Mach 10 flow around the ESA EXPERT atmospheric reentry vehicle
The EBF density, vibrational and rotational temperatures, and velocity in low-density hypersonic flows $\left(<10^{16}\right.$ molecules $\left./ \mathrm{cm}^{3}\right)$ on different species have been investigated by many researchers [1-11].

The technique has also been used on several occasions for in-flight measurements on board rockets to probe the shock layer or the upper atmosphere [5, 9]. Reference [8] is an excellent long review of the technique is based on the excitation and related fluorescence induced by an electron beam on the gas atoms and molecules along the electron beam path.

Figure 1 presents a typical setup of EBF in a wind tunnel application. In a low density gas flow, the use of an energetic electron beam (typically, $25 \mathrm{keV}$ ) induces excitations in gas all along the beam. These excitations produce fluorescence emitted at positions where collisions occur between electrons (at relativistic velocities) and molecules. Thus, EBF spectra usually range from X ray to the infrared. Each molecular or atomic species has its characteristic EBF spectral signature in the form of characteristic vibrational bands or rotational emission lines from which measurements specific to that specie can be performed.

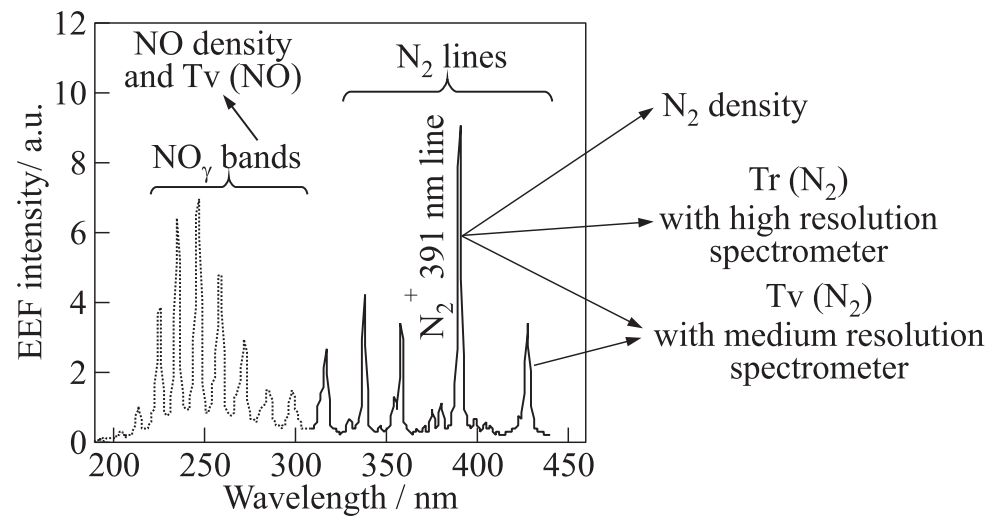

Figure 2 The EBF spectrum of $\mathrm{N}_{2}$ and NO 


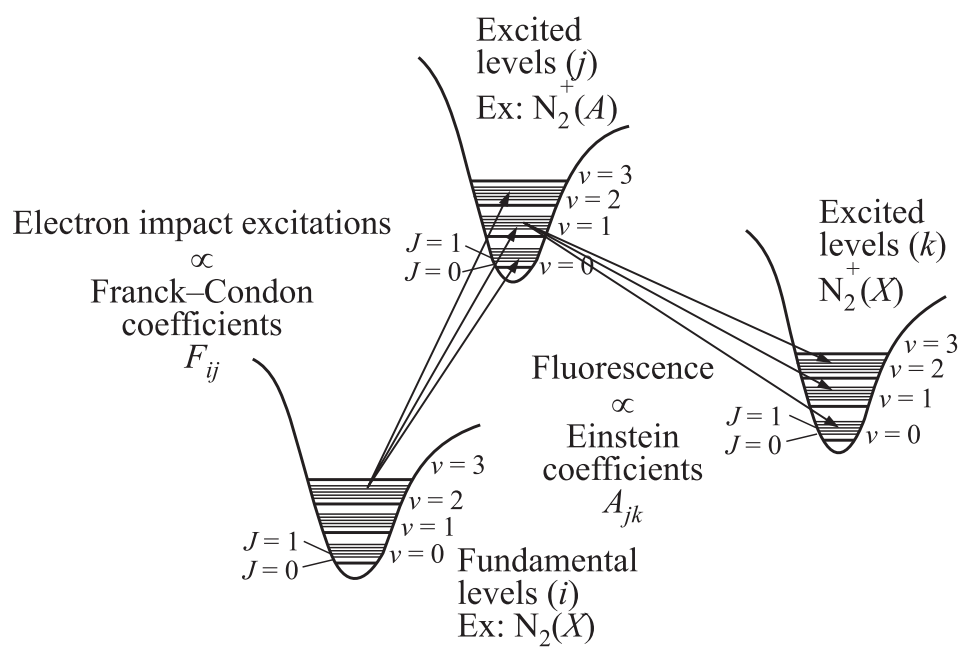

Figure 3 Electron beam excitation-fluorescence principle

For molecular nitrogen, the main emissions in the ultraviolet (UV) and visible spectrum (Fig. 2) are mainly the first negative system $\mathrm{N}_{2}^{+}(1 \mathrm{~N})$ and the second positive system $\mathrm{N}_{2}(2 \mathrm{P})$ from which most of the measurements are performed. For NO, the most prominent bands are the gamma bands in the UV between 200 and $300 \mathrm{~nm}$.

Moreover, the use of monoenergetic electrons allows one, from knowledge of the radiative emission and excitation coefficients (Fig. 3), to trace the intensity of a fluorescence line to the population number density of a fundamental energy level.

The intensity of a line is proportional to the total density of the probed specie. This linear dependence is valid up to density levels of $10^{16} \mathrm{~cm}^{-3}$. Above that value, there are two phenomena which cause deviations from linearity:

- fluorescence quenching (collisional nonradiative deexcitation); and

- supplementary excitations by secondary electrons (created through ionization from the electrons of the beam and which have sufficient energy to bring excitations (mainly, quadrupolar).

Beam dispersion becomes important also as from $10^{16} \mathrm{~cm}^{-3}$ which is, therefore, upper density limit for the EBF technique.

The EBF measurements can be useful in the following applications:

- validation of aerodynamic simulation codes from wind-tunnel or in-flight testing; 
Table 1 Parameters which can be measured by EBF on nitrogen-based flows

\begin{tabular}{ll}
\hline \multicolumn{1}{c}{ Parameter } & \multicolumn{1}{c}{ Range } \\
\hline Flow density & $10^{13}-10^{16}$ molecules $/ \mathrm{cm}^{3}$ \\
\hline Flow visualization & $10^{13}-10^{17}$ molecules $/ \mathrm{cm}^{3}$ \\
\hline $\begin{array}{l}\text { Temperatures } \\
- \text { of rotation } T_{r}\end{array}$ & $\begin{array}{l}\text { From a few to more } \\
\text { - of vibration } T_{v}\end{array}$ \\
\hline
\end{tabular}

- gas-surface accommodation; and

- atmospheres of other planets.

Table 1 presents the different parameters which can be measured on nitrogenbased flows.

\section{PROTOTYPE FOR IN-FLIGHT MEASUREMENTS}

The EBF technique is well known in ground facilities but has been seldom used for in-flight experiments due to its complexity and dimensions, particularly, for the electron gun.

ONERA has developed in the frame of one of its internal projects on hypersonic, a new concept of gun that associates small size, low power consumption and that does not require secondary vacuum to operate. These features are expected to greatly ease the in-flight application of EBF. The prototype of such a gun is illustrated in Fig. 4. Ions produced through an electrical discharge between the anode (wire) and the walls are accelerated towards the cathode maintained at a high voltage $(-25 \mathrm{keV})$. Their impacts against the cathode extract electrons which are accelerated counterwise and are collimated into a thin beam by the geometry of the system and provide a few milliampere of beam current. Operation with internal pressure up to $5 \mathrm{~Pa}$ of air has been demonstrated.

A single stage differential pumping with a small turbopump $(10 \mathrm{l} / \mathrm{s})$ and a 3 -millimeter conductance is able to maintain less than $3 \mathrm{~Pa}$ in the gun with more than $100 \mathrm{~Pa}$ outside.

Based on the advent of this new electron gun, ONERA recently carried a study aimed at designing, building, and validating a laboratory prototype to demonstrate the feasibility of obtaining a more compact EBF instrument capable of all the envisioned in-flight measurements $[7,11]$. The principle of the targeted prototype is shown in Fig. 5.

The EBF prototype will use an electron gun to produce a pulsed (around $10 \mathrm{~Hz}$ ) electron beam of about 25-kiloelectronvolt energy, about 1-milliampere 


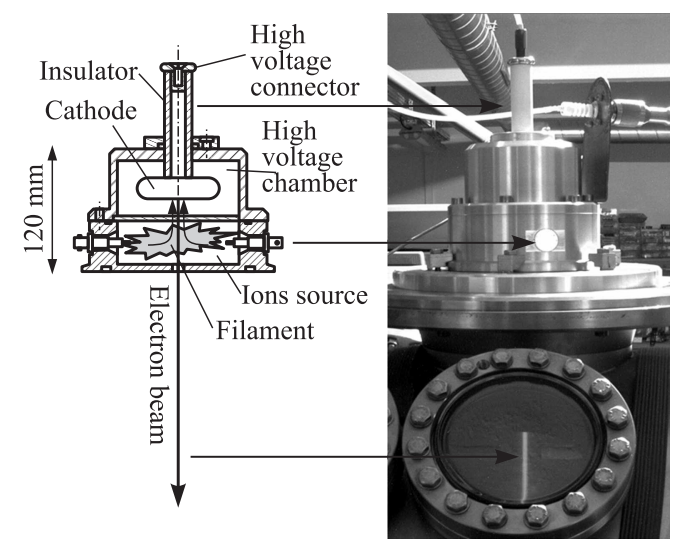

Figure 4 Miniature electron gun prototype

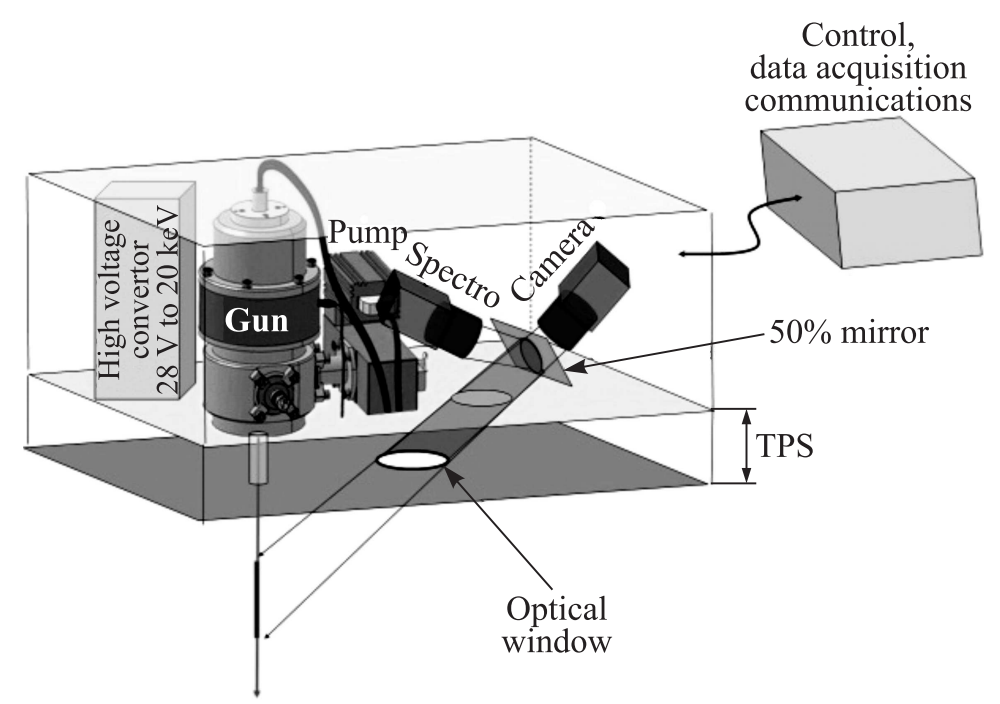

Figure 5 Target EBF assembly

intensity and 2-millimeter diameter which will be emitted through a small diameter (few millimeters) exit on the vehicle wall. A turbomolecular pump and highvoltage power converters operating from $24 \mathrm{~V}$ are needed for the beam emission. At least two optical detectors (one CCD (charge coupled device) camera and one spectrometer) will be used to observe, through one or two optical windows on the vehicle wall, the fluorescence induced by the electron beam. The data collected 
will be transmitted to the vehicle controller for saving and/or teletransmission. Processing of the data will be done during the postflight analysis.

The perimeter of development for the in-flight EBF system has been set to have at least the following measurement objectives:

- specie density: $\rho / \rho_{\propto}$ profile across a shock layer. The specie is $\mathrm{N}_{2}$ and eventually $\mathrm{NO}$ in case of an atmospheric reentry or $\mathrm{CO}$ and $\mathrm{CO}_{2}$ in case of a Martian reentry; and

- Temperature of vibration $T_{\mathrm{vib}}$ and/or temperature of rotation $T_{\text {rot }}$ of one of the above-mentioned species.

The measurements are to be performed along a line perpendicular to the wall of the vehicle. The segment of the line to be measured is of minimum length of $100 \mathrm{~mm}$ with the center at about $300 \mathrm{~mm}$ from wall of a vehicle. The measurements are to be provided at a minimum repetition rate of $10 \mathrm{~Hz}$ in the altitude range of 70 to $50 \mathrm{~km}$. Measurements at higher altitudes are possible with this technique but at the expense of lower signal-to-noise ratio which can partly be compensated by a longer integration time $(\sim 1 \mathrm{~s})$ and lower repetition rate $(\sim 1 \mathrm{~Hz})$. Measurements are less likely below $50 \mathrm{~km}$ due to nonlinearity in the fluorescence signal as well as high beam dispersion and attenuation

\section{LABORATORY PROTOTYPE}

These tests were carried in a vacuum chamber (cylinder $3.6 \mathrm{~m}$ long and of diameter $0.4 \mathrm{~m}$ ) with nearly all the key components of the EBF assembly positioned on a metallic plate of larger dimensions than a flight dedicated plate (Fig. 6a). Such a large plate is required for the coupling (to avoid vacuum leaks when coupling) to the vacuum chamber. Moreover, the vacuum chamber is not large enough to allow complete immersion of the EBF assembly which could, therefore, not be tested to operate as a whole instrument in vacuum conditions.

Figure $6 b$ shows the coupling of this intermediary setup to the vacuum chamber and the fluorescence trace of the electron beam during its operation.

The last validation tests of the final laboratory prototype are performed in a small transparent vacuum chamber. This chamber is a cylinder of diameter $300 \mathrm{~mm}$ and of length $400 \mathrm{~mm}$ with the cylindrical part made of glass of $8 \mathrm{~mm}$ thick. There are metallic plates at top and bottom of this cylinder. The EBF assembly base plate has been adapted to the top plate where appropriate holes have been drilled for the prism windows and electron beam exit. The bottom plate is equipped with necessary feed-troughs for pumping and pressure monitoring. Figure 6 shows the setup disposed on an office table. Aside the cylinder is placed an electronic box which has been specially manufactured to control manually the operation of the EBF assembly. This electronic box also encompass a 


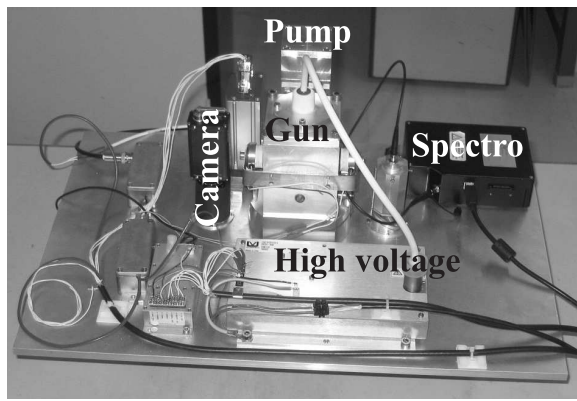

(a)

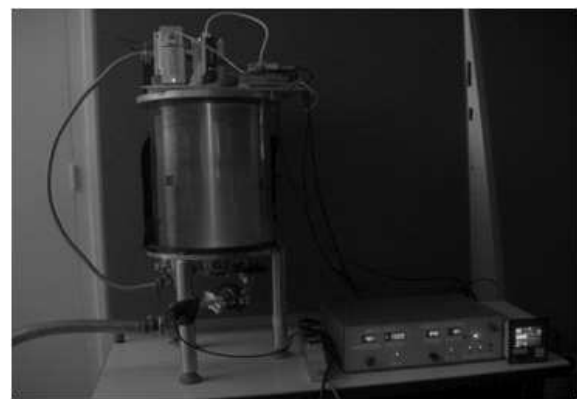

(b)

Figure 6 Tests of the intermediary EBF setup in a vacuum chamber: $(a)$ assembling of the intermediary EBF setup; (b) operation of the setup showing a fluorescent electron beam

converter to provide 24-volt power supply to the EBF prototype from standard 220 -volt power supply.

At the first step, the high voltage is limited to $15 \mathrm{keV}$ to avoid $\mathrm{X}$-ray emission crossing the glass wall of the cylinder. This result validates the correct operation of the EBF laboratory prototype.

The main objectives of the present study were to demonstrate the feasibility of manufacturing a compact EBF assembly. The next steps now are to adapt this assembly to comply with the harsh environmental requirements for a space flight. The laboratory prototype successfully validated as described above has been manufactured with many off-the-shelf components.

\section{EXPERIMENTAL DETAILS AND RESULTS}

A spectral analysis of different gases and their mixtures were performed in a vacuum chamber. The data from this spectral analysis will be used for postexperiment data reduction.

In the present experiment, a beam of $14 \mathrm{keV}$ induces fluorescence intensity as it penetrates the gas. The vacuum chamber was maintained at gas pressure between $10^{-3}$ and 1 mbar at a temperature of $300 \mathrm{~K}$ (static mode). A turbomolecular pump allows making differential pumping between the ion source and the rest of the vacuum chamber.

The fluorescent emission resulting from electron impact and ionization of the gas molecules could be observed through a UV-quartz window. A Faraday cup mounted to the vacuum chamber was used to collect the electrons after they passed through the chamber, and the current of the beam was thus recorded for each image or spectra collected. 


\subsection{Imaging Fluorescence}

A CCD camera system of $1340 \times 400$ pixel array was used to make a plane image of the electron beam. This camera allowed the beam dispersion study which consisted of direct measurement of the FWHM (full width at half maximum) of the beam compared to the axial distance.

A filter centered at $391.4 \mathrm{~nm}$ with a FWHM bandwidth of $10 \mathrm{~nm}$ was used to observe the most prominent transition of $\mathrm{N}_{2}, \mathrm{~B}^{2} \Sigma_{u}^{+} \rightarrow \mathrm{X}^{2} \Pi_{g}$. Images of the electron beam fluorescence were recorded at various pressures (0.1 to 1 mbar) and various beam voltages ( 15 to $27 \mathrm{kV}$ ). Each images was corrected from the background and the signal normalized by the beam current.

Figure 7 shows the beam dispersion after $30 \mathrm{~cm}$ of propagation. The axis originates at $30 \mathrm{~cm}$ corresponding to the output of the beam through the 1-millimeter diameter.

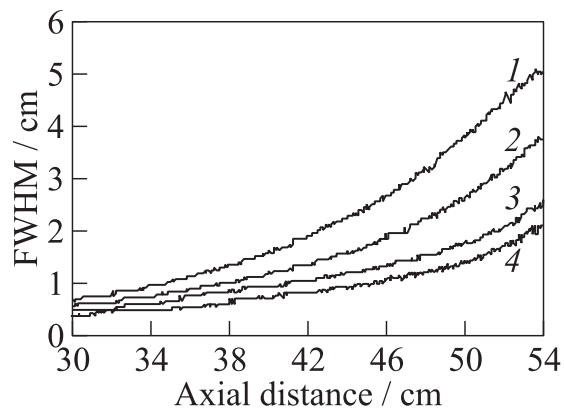

(a)

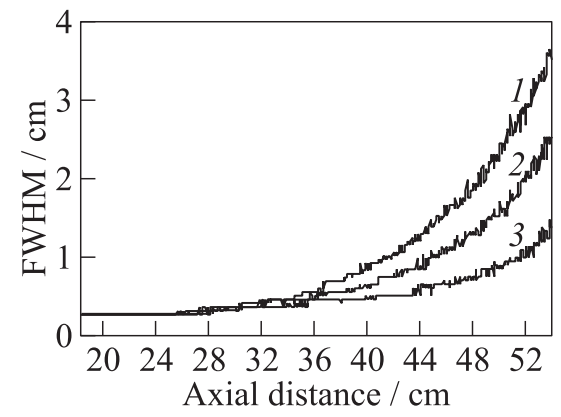

(b)

Figure 7 Electron beam dispersion at different pressures for 20-kiloelectronvolt electron beam energy $(1-0.7$ mbar; $2-0.5 ; 3-0.3$; and $4-0.14 \mathrm{mbar})$ and for 27-kiloelectronvolt electron beam energy $(1-0.6 \mathrm{mbar} ; 2-0.4$; and $3-0.2 \mathrm{mbar})$

\subsection{Spectroscopy}

A study of electronic spectra of some molecular gases was performed. This study enables to understand the chemical and physical processes that take place in studied atmospheres.

Depending of the interested region, various spectrometers and various gratings are used:

- Ocean Optics HR4000 (200 to $1100 \mathrm{~nm}$ and 0.2-nanometer resolution);

- BWTEC spectrometer from 200 to $600 \mathrm{~nm}$; and 


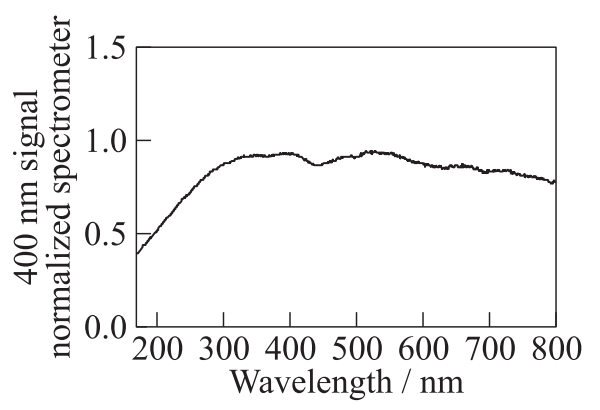

Figure 8 ACTON Princeton 2400 grooves $/ \mathrm{mm}$ grating normalized response in the region of $170-800 \mathrm{~nm}$. Wavelength correction by calibration with a Deuterium lamp

- Princeton's ACTON Instruments spectrometer with 300 grooves $/ \mathrm{mm}$ (1-nanometer resolution) to 2400 grooves/mm grating (0.125-nanometer resolution) covering 175 to $1300 \mathrm{~nm}$.

Spectroscopic investigations are followed by postdata reduction. Each spectrum was corrected by spectral radiance of the Deuterium lamp as a function of wavelength. Figure 8 shows the response of the ACTON spectrometer used for data correction with wavelength calibration using a deuterium lamp and background subtracted from collected spectrum.

Figures 9 and 10 show some of spectra for these gases and their mixtures:

- Fig. 9 shows spectra of $\mathrm{N}_{2} / \mathrm{NO}$ mixture gas at a pressure of 0.4 mbar. Vibrational bands between 300 and $450 \mathrm{~nm}$ corresponding to nitrogen bands $\left(\mathrm{N}_{2} 1 \mathrm{~N}\right.$ and $\left.\mathrm{N}_{2} 2 \mathrm{P}\right)$ and gamma bands of NO between 200 and $300 \mathrm{bm}$. Using 2400 grooves/mm grating, the rotational structure can be resolved; and

- Fig. 10 shows rotational structure of the first negative system of $\mathrm{N}_{2}$ $\left(\mathrm{B}^{2} \Sigma_{u}^{+} \rightarrow \mathrm{X}^{2} \Pi_{g}\right)$.

In Fig. 11, transition between 190 and $300 \mathrm{~nm}$ corresponds to B-X (first negative system) bands of $\mathrm{CO}^{+}$and comet-tail system of $\mathrm{CO}^{+}$between 300 and $500 \mathrm{~nm}$.

Figure 12 shows both $\mathrm{CO}_{2}^{+}, \mathrm{B}^{2} \Sigma_{u}^{+} \rightarrow \mathrm{X}^{2} \Pi_{g}$ doublet at $289 \mathrm{~nm}$ and the $\mathrm{CO}_{2}^{+}$, $\mathrm{A}^{2} \Pi_{u} \rightarrow \mathrm{X}^{2} \Pi_{g}$ between 337 and $450 \mathrm{~nm}$.

First attempt to identify some systems in a $\mathrm{N}_{2} / \mathrm{CO} / \mathrm{CO}_{2}$ mixture gas is shown in Fig. 13. 


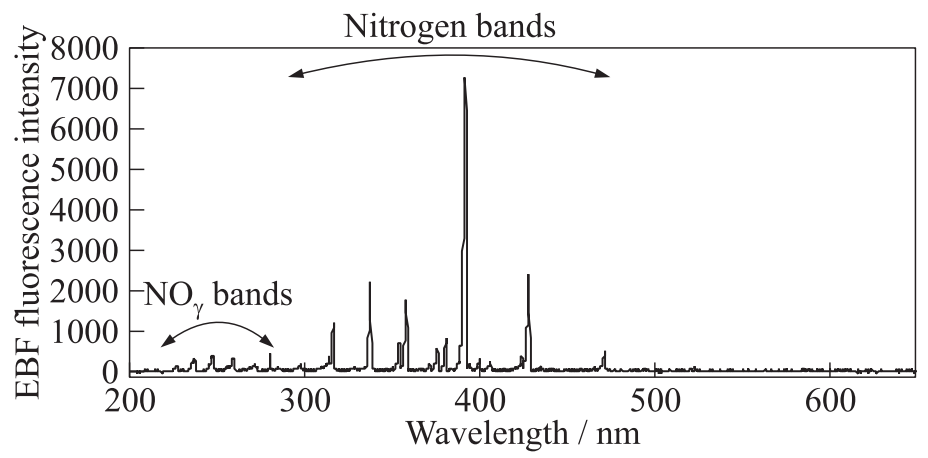

Figure 9 Spectrum of a $\mathrm{N}_{2} / \mathrm{NO}(99 \% / 1 \%)$ mixture gas at $0.4 \mathrm{mbar}$

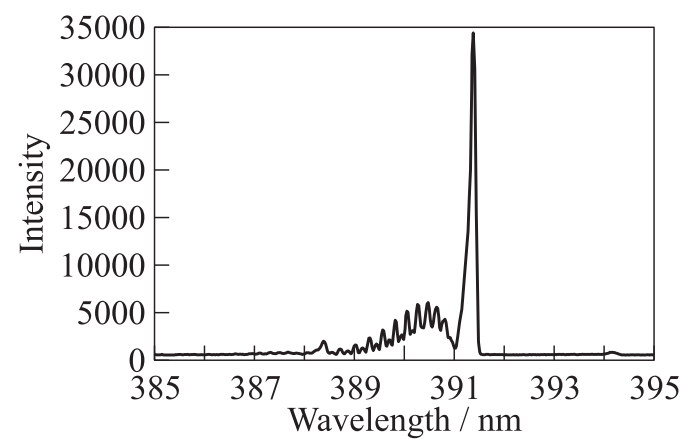

Figure 10 Rotational structure of first negative system of $\mathrm{N}_{2}$ corresponding to vibrational transition $\mathrm{N}_{2}, \mathrm{~B}^{2} \Sigma_{u}^{+} \rightarrow \mathrm{X}^{2} \Pi_{g}\left(\mathrm{~N}_{2} 1 \mathrm{~N}(0,0)\right.$

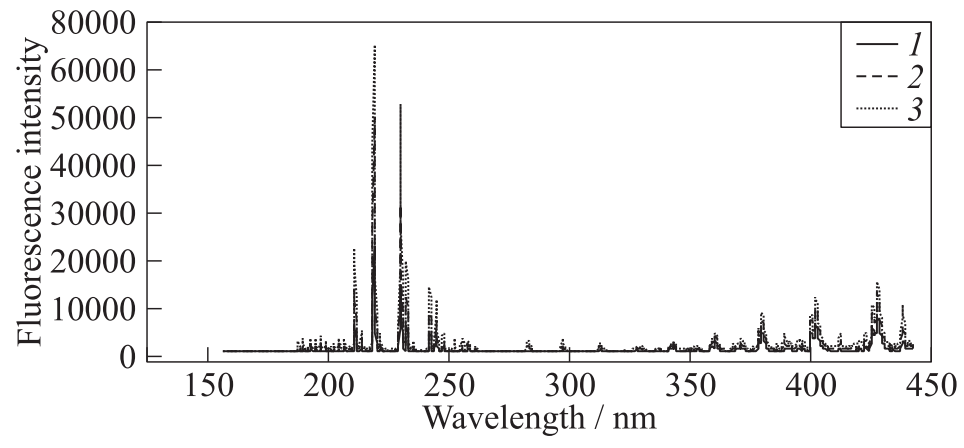

Figure 11 Spectrum of CO pure gas at $14.84 \mathrm{kV}$ and different gas pressures: 1 0.008 Torr; $2-0.016$; and $3-0.03$ Torr 


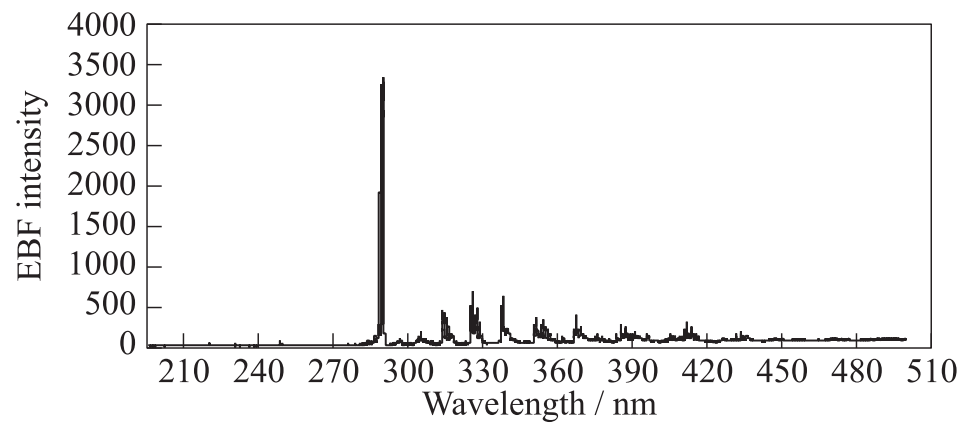

Figure 12 Spectrum of $\mathrm{CO}_{2}$ pure gas at $0.382 \mathrm{mbar}$

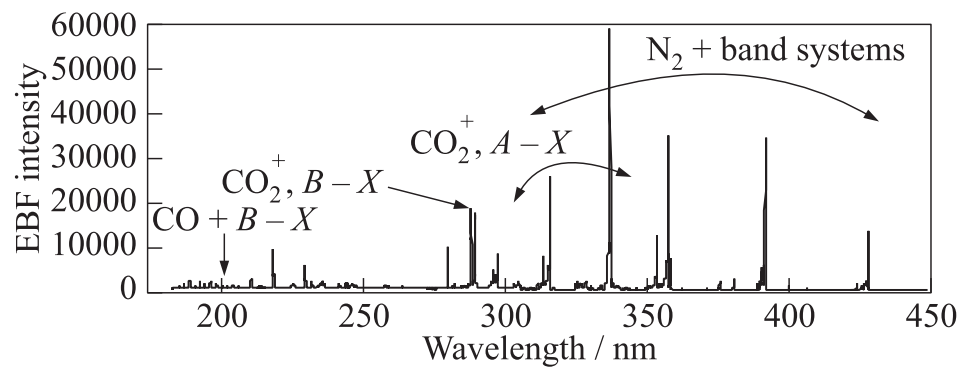

Figure 13 Spectrum of a mixture of $\mathrm{N}_{2} / \mathrm{CO} / \mathrm{CO}_{2}(85 \% / 10.5 \% / 4.5 \%)$

\section{CONCLUDING REMARKS}

A compact EBF laboratory prototype has been developed in view of in-flight measurements aboard a reentry demonstrator. The in-flight objectives are the same as for ground facilities: simultaneous and multipoint measurements of density, rotational and vibrational temperatures of $\mathrm{N}_{2}$ as well as density of NO. Other species like $\mathrm{CO}, \mathrm{CO}_{2}, \mathrm{He}$, and $\mathrm{Ar}$ are also possible with this technique provided that the observations are performed at wavelengths corresponding to these species.

The compact assembly encompasses all the main key components of the EBF technique (electron gun, turbopump, high-voltage converters, camera, and spectrometer) in a box of $375 \times 300 \times 250 \mathrm{~mm}$ and weighting about $11 \mathrm{~kg}$ with only few electrical interfaces. Most of the components have also a potential to sustain mechanical vibration and shock up to $20 \mathrm{~g}$ but here further qualification in a specialised facility is needed.

The laboratory prototype has been validated to emit a well collimated electron beam over a distance of at least $300 \mathrm{~mm}$ in a vacuum chamber at pressures 
up to $100 \mathrm{~Pa}$ and provided reference images and spectral acquisitions for density profile and $T_{v}$ temperature measurements. Spectra of in-flight interesting gases $\mathrm{N}_{2}, \mathrm{NO}, \mathrm{CO}$, and $\mathrm{CO}_{2}$ have been recorded, the data reduction codes are being developed, and a simulation-inversion program will enable to measure temperature and density parameters.

\section{ACKNOWLEDGMENTS}

The work has been sponsored by ESA.

\section{REFERENCES}

1. Herzberg, G. 1945. Infrared and Raman spectra of polyatomic molecules. Molecular spectra and molecular structure ser. Vol. II. Princeton, N. Jersey: Van Nostrand.

2. Nicholls, R. W. 1962. Franck-Condon factors and $r$-centroids to high vibrational quantum numbers for three bands systems of $\mathrm{CO}^{+}$and absolute band strengths for the comet-tail system. Can. J. Phys. 40:1772.

3. Muntz, E. P. 1968. The electron beam fluorescence technique. AGARDograph 132.

4. Krupenie, P. H., and W. Benesch. 1968. Electronic transition moment integral for the first ionization of $\mathrm{CO}$ and the A-X transition of $\mathrm{CO}^{+}$. J. Res. Nat. Stand. Sect. A 72:495.

5. De Leeuw, J.H., and W.E.R. Davies. 1972. Measurement of temperature and density in the upper atmosphere using an electron beam. Can. J. Phys. 50(10):1044.

6. Cattolica, R. J., R. J. Schmitt, and R.E. Palmer. 1990. Feasibility of non-intrusive optical diagnostic measurements in hypersonic boundary layers for flight experiments. AIAA Paper No. 90-0627.

7. Mohamed, A.K., T. Pot, and B. Chanetz. 1995. Diagnostics by electron beam fluorescence in hypersonics. ICIASF'95 Record 95-CH3482-7,14.1, International Congress on Instrumentation in Aerospace Simulation Facilities. Wright-Patterson AFB, Ohio, USA.

8. Gochberg, L. A. 1997. Electron beam fluorescence methods in hypersonic aerothermodynamics. Prog. Aerospace Sci. 33:431-80.

9. Kawashima, T., K. Oyama, and K. Suzuki. 1999. A measurement of vibrationalrotational temperature and density of molecular nitrogen in the upper atmosphere by rocket-borne electron beam induced luminescence. Report No.672. Institute of Space and Astronautical Science, Japan.

10. Mohamed, A., J. Bonnet, J.-P. Faléni, U. Koch , B. Esser, A. Ghulan, R. Janovsky, and P. Vahlenkamp. 2007. ESA ITI Project - demonstration of electron beam fluorescence (EBF) feasibility for flight application in the DLR LBK wind tunnel. ONERA Report RF 1/10453 DMPH.

11. Mohamed, A. J. Bonnet, J.-P. Faléni, and T. Schmid. 2008. Electron beam fluorescence (EBF) technique for ATD applications. TN 1.2. EBF-TS. Technical specifications. ONERA Report RT 2/12105 DMPH. 